\title{
Photon indistinguishability measurements under pulsed and continuous excitation
}

\author{
Ross C. Schofield $\odot,{ }^{1}$ Chloe Clear $\odot,{ }^{2}$ Rowan A. Hoggarth $\odot,{ }^{1}$ Kyle D. Major $\odot,{ }^{1}$ \\ Dara P. S. McCutcheon $\odot,^{2}$ and Alex S. Clark $\oplus^{1, *}$ \\ ${ }^{1}$ Centre for Cold Matter, Blackett Laboratory, Imperial College London, Prince Consort Road, London, SW7 2AZ, United Kingdom \\ ${ }^{2}$ Quantum Engineering Technology Labs, H. H. Wills Physics Laboratory and Department of Electrical and Electronic Engineering, \\ University of Bristol, Bristol, BS8 1FD, United Kingdom
}

(Received 26 March 2021; accepted 23 December 2021; published 18 January 2022)

\begin{abstract}
The indistinguishability of successively generated photons from a single quantum emitter is most commonly measured using two-photon interference at a beam splitter. Whilst for sources excited in the pulsed regime the measured bunching of photons reflects the full wave-packet indistinguishability of the emitted photons, for continuous wave (cw) excitation, the inevitable dependence on detector timing resolution and driving strength obscures the underlying photon interference process. Here we derive a method to extract full photon wave-packet indistinguishability from $\mathrm{cw}$ measurements by considering the relevant correlation functions. The equivalence of both methods is experimentally verified through a comparison of cw and pulsed excitation measurements on an archetypal source of photons, a single molecule.
\end{abstract}

DOI: 10.1103/PhysRevResearch.4.013037

\section{INTRODUCTION}

Many photonic quantum technologies rely on the quantum interference of photons, including linear optical quantum information processing [1], cluster state generation [2], boson sampling [3], quantum metrology [4], and Bell-state measurements in quantum communication [5], and teleportation schemes [6]. However, this quantum interference is only possible if the photons used are quantum mechanically indistinguishable, and it is therefore paramount when developing a single photon source that the indistinguishability of emitted photons is quantified. While this can in principle be inferred through separate characterization of the photons' polarization, spatial, temporal, and frequency modes, a more rigorous method which directly proves their usefulness is to measure the two-photon interference effect itself.

This two-photon interference effect was first seen in an interference pattern by Ghosh and Mandel [7], followed by Hong, Ou and Mandel showing interference at a beamsplitter [8], both using photons probabilistically generated through spontaneous parametric down-conversion of a pump laser in a nonlinear crystal. Since then routes toward generating photons on-demand have emerged [9], for example those using single quantum emitters such as atoms [10], quantum dots [11-14], crystalline defects [15] and single molecules [16]. For these systems it is common to interfere successively emitted photons from a single source by introducing an appropriate delay

\footnotetext{
*alex.clark@imperial.ac.uk

Published by the American Physical Society under the terms of the Creative Commons Attribution 4.0 International license. Further distribution of this work must maintain attribution to the author(s) and the published article's title, journal citation, and DOI.
}

and mixing the two signals on a beam splitter. The interference is then manifest as a reduction in coincidence counts at the beam splitter outputs, as measured by the second-order correlation function $g^{(2)}(\tau)$.

If the emitter is excited regularly with a pulsed laser, then the normalized time-integrated difference between $g^{(2)}(\tau)$ measurements for photons input with perpendicular and parallel polarization directly gives the full photon wave-packet indistinguishability $\mathcal{I}=\left\langle\psi_{1} \mid \psi_{2}\right\rangle$, where $\left|\psi_{1,2}\right\rangle$ represent the quantum states, or wave functions, of the two interfering photons at the point of measurement. This reflects the underlying modal purity of the photons and gives the probability of two photon interference, sometimes called the coalescence probability $[11,17]$. This probability is independent of any temporal post-selection or detector timing response.

On the other hand, source excitation with a continuous wave (cw) laser is also commonly used [18-21], and the timeresolved $g^{(2)}(\tau)$ is credited with indicating the extent of the two-photon interference phenomenon. These measurements generally measure the visibility of the interference effect. However, visibility is inconsistently defined and frequently limited to commenting on the photon interference at zero time delay. The value of $g^{(2)}(0)$ is highly dependent on detector timing resolution and tends to zero for perfect detector resolution regardless of the photon spectral purity [22], as the measurement itself is effectively a frequency filter. This metric does not account for spectral purity and is dependent on detector timing response, meaning it cannot directly correspond to full photon wave-packet indistinguishability. While methods to extract detector resolution independent metrics from $\mathrm{cw}$ measurements have been proposed [23], they do not give the unitless indistinguishability measure found in the pulsed case.

In this paper, we derive correlation functions for both pulsed and cw excitation of a single photon emitter and develop a method to determine the full photon wave-packet 
indistinguishability under $\mathrm{cw}$ excitation, as opposed to just stating the visibility at $\tau=0$ as is currently performed. Our method also takes into account the dependence of the measurement on driving strength. This is experimentally verified through measurements of a single dibenzoterrylene (DBT) molecule in an anthracene host matrix. Pulsed and cW measurements are performed on the same molecule to independently determine $\mathcal{I}$, showing the correspondence of the two methods. This equivalence provides a useful analysis tool for developing on-demand photon sources from single quantum emitters.

\section{THEORY}

To begin we consider a beam splitter with successively generated photons from a single quantum emitter at its inputs. The derivation of the unnormalized second-order correlation function of photons from a single emitter measured at the outputs of this beam splitter is outlined in Appendix A. For parallel polarized input photons with positive electric field operator $E(t)$, this is

$$
\begin{aligned}
G_{\|}^{(2)}(\tau, t)= & \frac{1}{2}\left(4 G_{\mathrm{HBT}}^{(2)}(t, \tau)-\|\left\langle E^{\dagger}(t+\tau) E(t)\right\rangle^{2}\right. \\
& \left.+\left\langle E^{\dagger}(t) E(t)\right\rangle\left\langle E^{\dagger}(t+\tau) E(t+\tau)\right\rangle\right),
\end{aligned}
$$

where $G_{\mathrm{HBT}}^{(2)}(t, \tau)=\frac{1}{4}\left\langle E^{\dagger}(t) E^{\dagger}(t+\tau) E(t+\tau) E(t)\right\rangle$ is the Hanbury Brown and Twiss second-order correlation function, relating to the case whereby only one input field is incident on the beam splitter.

For the case of pulsed off-resonant excitation, we model the emitter as a two-level system initially populated in its excited state, with dipole operator $\sigma=|g\rangle\langle e|$, with $|e\rangle$ and $|g\rangle$ the excited and ground states. We integrate Eq. (1) over $t$ to give a coincidence probability per pulse [24], to find

$$
G_{\|}^{(2)}(\tau)=\int_{0}^{\infty} d t\left[P_{e}(t) P_{e}(t+\tau)-\left|g^{(1)}(t+\tau, t)\right|^{2}\right],
$$

where the first order-correlation function is $g^{(1)}\left(t_{1}, t_{2}\right)=$ $\left\langle\sigma^{\dagger}\left(t_{1}\right) \sigma\left(t_{2}\right)\right\rangle$, the excited state population at time $t$ is $P_{e}(t)=$ $\left\langle\sigma_{e e}(t)\right\rangle$ with $\sigma_{e e}=\sigma^{\dagger} \sigma$ and the input electric field operators are set to the dipole operators to capture the two-level system. Under pulsed excitation $G_{\mathrm{HBT}}^{(2)}(t, \tau)=0$, as $\sigma^{2}=0$ [25]. For perpendicular input polarizations photon distinguishability is imposed, and the coincidence probability becomes

$$
G_{\perp}^{(2)}(\tau)=\int_{0}^{\infty} d t P_{e}(t) P_{e}(t+\tau)
$$

where the interference term goes to zero. The photon indistinguishability is defined as the normalized difference in coincidence events for parallel and perpendicular input polarizations for photons arriving simultaneously, integrated over all detection time differences $\tau$ [26]:

$$
\mathcal{I}=\frac{\int d \tau G_{\perp}^{(2)}(\tau)-\int d \tau G_{\|}^{(2)}(\tau)}{\int d \tau G_{\perp}^{(2)}(\tau)} .
$$

For the case of a quantum emitter with spontaneous decay rate $\Gamma_{1}$ and dephasing rate $\Gamma_{2}=\Gamma_{1} / 2+\gamma$ where $\gamma$ represents some pure dephasing, we find $\mathcal{I}=\Gamma_{1} /\left(2 \Gamma_{2}\right)$, see Supplemental Material for further details [28].
Under nonresonant $\mathrm{cw}$ excitation conditions, Eq. (1) is evaluated in its steady state by taking $t \rightarrow \infty$ giving the measured coincidences for parallel inputs to be

$$
g_{\|}^{(2)}(\tau)=\frac{1}{2}+\lim _{t \rightarrow \infty} \frac{g^{(2)}(t, t+\tau)-\left|g^{(1)}(t+\tau, t)\right|^{2}}{2 P_{e}^{2}},
$$

which in this case is normalized by the square of the excited steady-state population $P_{e}=\lim _{t \rightarrow \infty} P_{e}(t)$ and we have defined $g^{(2)}\left(t_{1}, t_{2}\right)=\left\langle\sigma^{\dagger}\left(t_{1}\right) \sigma^{\dagger}\left(t_{2}\right) \sigma\left(t_{2}\right) \sigma\left(t_{1}\right)\right\rangle$ [25]. For the case of perpendicular inputs where the fields can be treated as uncorrelated, we have

$$
g_{\perp}^{(2)}(\tau)=\frac{1}{2}+\lim _{t \rightarrow \infty} \frac{g^{(2)}(t, t+\tau)}{2 P_{e}^{2}} .
$$

It is common to consider a reduction in $g_{\|}^{(2)}(\tau)$ at $\tau=0$ as an indication of the probability of two-photon interference and photon purity. However, since $\sigma(t)^{2}=0$, it follows that $g^{(2)}(t, t)=0$, while $g^{(1)}(t, t)=P_{e}(t)$, and one can therefore see from Eq. (5) that $g_{\|}^{(2)}(0)=0$ regardless of the photon coherence. In experiments, deviations from this value arise due to detector imperfections being unable to precisely resolve $\tau=0$. As such the value of $g_{\|}^{(2)}(0)$ at best reflects a combination of the detector response and photon distinguishability. We could, perhaps, integrate over $\tau$ as in the pulsed case, but as these cw quantities give coincidences per unit time and the system is driven, the time-integrals diverge. To overcome this, we propose to first subtract the excited steady-state population which recovers a convergent integral similar to that in Eq. (4), which after cancellations becomes

$$
\tilde{\mathcal{I}}(S)=\frac{\int d \tau\left[1-g_{\|}^{(2)}(\tau)\right]-\int d \tau\left[1-g_{\perp}^{(2)}(\tau)\right]}{\int d \tau\left[1-g_{\perp}^{(2)}(\tau)\right]},
$$

which in general is a function of the cw driving strength described by the saturation parameter $S$. Our crucial observation is that in the limit of weak driving $\tilde{\mathcal{I}}(0)=\mathcal{I}$, and we see that $\mathrm{cw}$ measurement contains the true photon indistinguishability that we seek.

It is not, of course, possible to measure the correlation function at $S=0$ as no photons are emitted. We therefore seek an analytical expression for $\tilde{I}(S)$, from which $\mathcal{I}$ can be extracted. To do so we consider an incoherently driven effective two-level system, obtained by adiabatically eliminating a fast decaying higher energy state used for off-resonant excitation. The adiabatic elimination is valid provided decay from the pump level at a rate $\beta$ is fast compared to the other system rates $\left(\beta \gg S \Gamma_{1}, \Gamma_{2}\right)$, see Appendix B for details.

The result is a second-order Born-Markov master equation for the effective two-level system density operator $\rho$ :

$$
\partial_{t} \rho(t)=\Gamma_{1}\left(\mathcal{L}_{\sigma}[\rho(t)]+S \mathcal{L}_{\sigma^{\dagger}}[\rho(t)]\right)+2 \gamma \mathcal{L}_{\sigma_{e e}}[\rho(t)],
$$

where $\mathcal{L}_{X}[\rho(t)]=X \rho(t) X^{\dagger}-\frac{1}{2}\left\{X^{\dagger} X, \rho(t)\right\}$ is a Lindblad operator which captures open quantum system dissipators. The first dissipator describes the spontaneous emission of the emitter. The incoherent driving is captured by the term involving the saturation parameter $S=\Omega^{2} /\left(\beta \Gamma_{1}\right)$ where $\Omega$ is the Rabi frequency between the ground and higher energy pump level. The final dissipator represents the pure dephasing of the emitter with rate $\gamma$. Using this master equation and the quantum 
regression theorem [27], we find the correlation function for parallel polarization alignment to be

$$
g_{\|}^{(2)}(\tau)=1-\frac{\mathcal{V}}{2} e^{-\Gamma_{1}(1+S)|\tau|}\left(1+\mathcal{M} e^{-2 \gamma|\tau|}\right),
$$

where we have introduced $\mathcal{V}$ to account for any imperfection in anti-bunching visibility and $\mathcal{M}$ to account for any modal distinguishability with no temporal dependence on the timescale of the detector timing resolution, such as incoherent sideband emission or polarization mismatch. For perpendicular polarization $g_{\perp}^{(2)}(\tau)$ is given by Eq. (9) with $\mathcal{M}=0$. Using these in Eq. (7) we find

$$
\tilde{\mathcal{I}}(S)=\mathcal{M} \frac{\Gamma_{1}(1+S)}{\Gamma_{1}(1+S)+2 \gamma},
$$

which allows for cw measurements of $g_{\| / \perp}^{(2)}(\tau)$ to be integrated at a known $S$ and extrapolated to $S=0$ to give $\mathcal{I}$. The effective two-level system model from which Eq. (10) is derived holds for $\beta \gg S \Gamma_{1}$, which is well within the validity of our system parameters (see Ref. [28] ). At high $S$ stimulated emission from $S_{1, n>0}$ leads to deviations from the behavior described in Eq. (8) [29].

\section{EXPERIMENT AND RESULTS}

We now turn to indistinguishability measurements of photons emitted by a single DBT molecule to verify our theory. To isolate a single molecule we used a DBT-doped anthracene nanocrystal grown using a reprecipitation technique [30], see Fig. 1(a). This crystal was deposited onto a gold-coated silicon substrate with a $85 \mathrm{~nm}$ silica spacer layer and protected with a 200-nm-thick layer of PVA. The gold mirror increases the collection efficiency of light from the molecule [31]. The sample was cooled to $4.7 \mathrm{~K}$ in a closed-cycle cryostat (Montana Cryostation) that forms part of a confocal microscope shown in Fig. 1(a). A nanocrystal was selected and illuminated with a cw Ti:Sapphire laser (MSquared, SolsTiS), directed using the scanning mirrors. Figure 1(b) shows the energy level diagram of a DBT molecule and the laser frequencies used for excitation. The laser was tuned in frequency to identify a molecule resonance through excitation of the $S_{0,0} \rightarrow S_{1,0}$ zero-phonon line (ZPL) transition, around $784 \mathrm{~nm}$, while the red-shifted fluorescence (>790 nm), shown in Fig. 1(c), from the $S_{1,0} \rightarrow S_{0, n>0}$ transitions was collected in a multi-mode fiber and detected with a silicon avalanche photodiode (APD).

A single DBT resonance was found at $784.45 \mathrm{~nm}$, and initial characterization was performed by repeating scans at increasing excitation powers to determine the maximum count rate and linewidth $\Delta v$ of the molecule at each power. This was used to determine the dephasing rate $\Gamma_{2}$ and saturation behavior of the molecule using the power-broadening relationship [32]

$$
\Delta v=\frac{\Gamma_{2}}{\pi} \sqrt{1+S} .
$$

From this, we find $\Gamma_{2}=2 \pi \times 35(4) \mathrm{MHz}$.

The $\mathrm{cw}$ laser was then tuned to $766.67 \mathrm{~nm}$ resonant with a $S_{0,0} \rightarrow S_{1, n>0}$ transition, shown as a blue arrow in Fig. 1(b). The collection was changed to use a single mode fiber and a narrowband $(0.15 \mathrm{~nm})$ tunable reflective notch filter po-
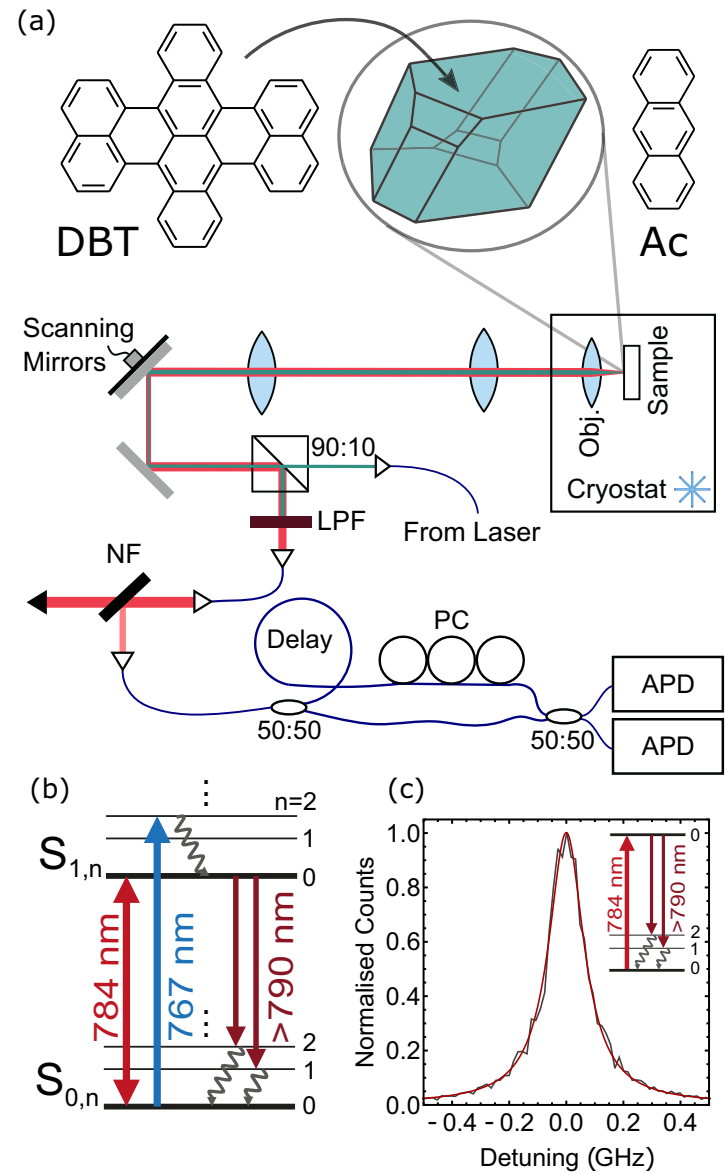

FIG. 1. (a) Schematic of the nanocrystal and experimental apparatus used. Diagrams of dibenzoterrylene (DBT), anthracene (Ac) and a DBT containing Ac nanocrystal are shown. In the simplified experimental apparatus, blue lines are single mode fibers, green is the excitation laser, dark red is all molecular emission and light red is ZPL emission. The confocal microscope setup consists of; 90:10: 90\% reflection, $10 \%$ transmission beam splitter; Obj.: Objective lens; LPF: long-pass filter; NF: notch reflection filter. The interferometer consists of; 50:50: 50\% reflection, 50\% transmission single mode fiber beam splitter; PC: fiber polarization controller; APD: avalanche photodiode. (b) Energy level diagram of the DBT molecule used showing the two electronic energy levels and the associated vibrational levels. Approximate wavelengths of the transitions are shown. (c) Fluorescence excitation spectrum of the DBT molecule ZPL showing the change in detected photon counts as the laser is tuned relative to the $S_{0,0} \rightarrow S_{1,0}$ transition frequency of the molecule. Data (grey) are fit with a Lorentzian (red) to obtain the linewidth. (Inset) Energy levels showing the excitation $(784 \mathrm{~nm})$ and collected fluorescence $(>790 \mathrm{~nm}$ ) wavelengths.

sitioned before the APDs. The filter response function and the expected effect on the molecule spectrum is shown in Ref. [28]. Only the coherent emission from the $S_{1,0} \rightarrow S_{0,0}$ ZPL transition will provide measurable interference; the narrow band filter is used to remove emission from the phonon sideband [31] and $S_{1,0} \rightarrow S_{0, n>0}$ transitions. After filtering we expect a ratio of coherent to total collected emission of $>99 \%$.

To verify single photon emission a Hanbury Brown and Twiss $g^{(2)}(\tau)$ measurement was performed, shown in Fig. 2, by splitting the fluorescence directly on a 50:50 beam splitter 


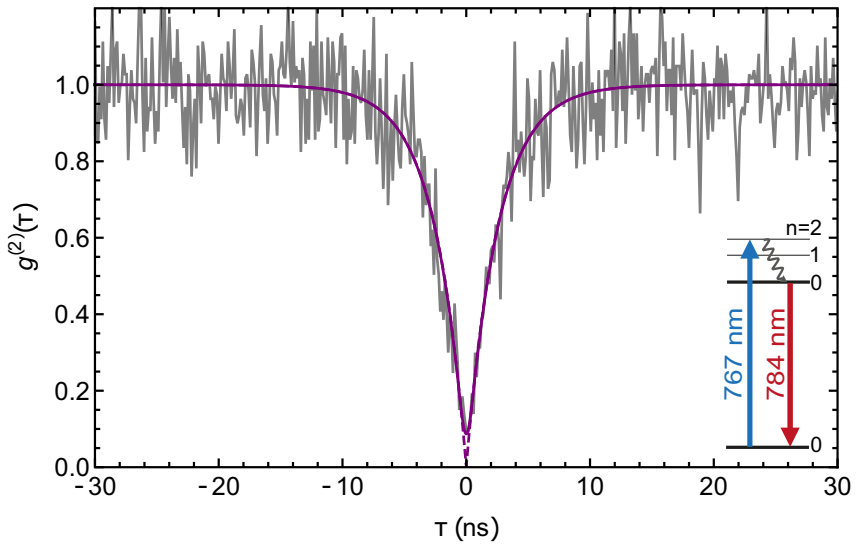

FIG. 2. A cw intensity correlation $g^{(2)}(\tau)$. Experimental data is in black, with theoretical fit using Eq. (12) with (solid) and without (dashed) accounting for detector response. After correction for timing jitter we find a visibility of $\mathcal{V}=1.00_{-0.03}^{+0}$, indicating a single emitter is being probed. (Inset) Energy levels showing the excitation (767 nm) and collected fluorescence (784 nm) wavelengths.

before two APDs. Fitting the data using [32]

$$
g^{(2)}(\tau)=1-\mathcal{V} e^{-\Gamma_{1}(1+S)|\tau|},
$$

we find a visibility of $\mathcal{V}=0.98_{-0.03}^{+0.02}$, which when accounting for detector timing jitter gives $\mathcal{V}=1.00_{-0.03}^{+0}$ indicating we are observing a single emitter. Accounting for the measured saturation parameter $S$ (see Ref. [28]) we find a population decay rate of $\Gamma_{1}=2 \pi \times 40$ (2) MHz. This is independently verified using a time-correlated single photon counting measurement with a pulsed Ti:sapphire laser (Coherent, Tsunami) which gives $\Gamma_{1}=2 \pi \times 39(3) \mathrm{MHz}$. Comparison of the dephasing and population decay rates gives $\Gamma_{1} / 2 \Gamma_{2}=0.57 \pm$ 0.09 , typical at these temperatures due to the excess thermal dephasing [31,32].

Turning now to measuring indistinguishability using $\mathrm{cw}$ two-photon interference, the fluorescence was sent to the fiber-based interferometer shown in Fig. 1(a). A 50:50 fiber beam splitter and delay fiber was used to temporally overlap photons at a second beam splitter, where two-photon interference occurs. A fiber polarization controller allowed for measurements of photons with parallel or perpendicular polarization.

The results of the parallel and perpendicular interference measurements at $S=1.3 \pm 0.1$ are shown in Figs. 3(a) and $3(\mathrm{~b})$. The data fall below 0.5 in the parallel case due to photon interference and coalescence. The side dips arise from antibunching at different time delays due to the different combinations of possible optical paths [20]. Fitting these side dips determines the $S$ and $\mathcal{V}$ parameters. Equation (9), convolved with the detector response function, is plotted over the data using the determined experimental parameters and $\mathcal{M}=0.98$, showing a good correspondence between the measurement and expected result. The nonconvolved function is shown as a dashed line. This is repeated for the orthogonal polarization $g_{\perp}^{(2)}(\tau)$ measurement, shown in Fig. 3(b), where $\mathcal{M}=0.04$. For a measurement with perfectly orthogonal polarization $\mathcal{M}=0$, however polarization drift during measurement resulted in a small two-photon interference contribution. This is characterized in Ref. [28]. Parallel and perpendicular interference measurements for $S=4.4 \pm 0.2$ are shown in Figs. 3(c) and 3(d). The difference between the theoretical curves in Fig. 3(c) at $\tau=0$ show the large effect the detector response has on using $g_{\|}^{(2)}(0)$ as a measure of indistinguishability. Although detector jitter affects this value, it does not affect the
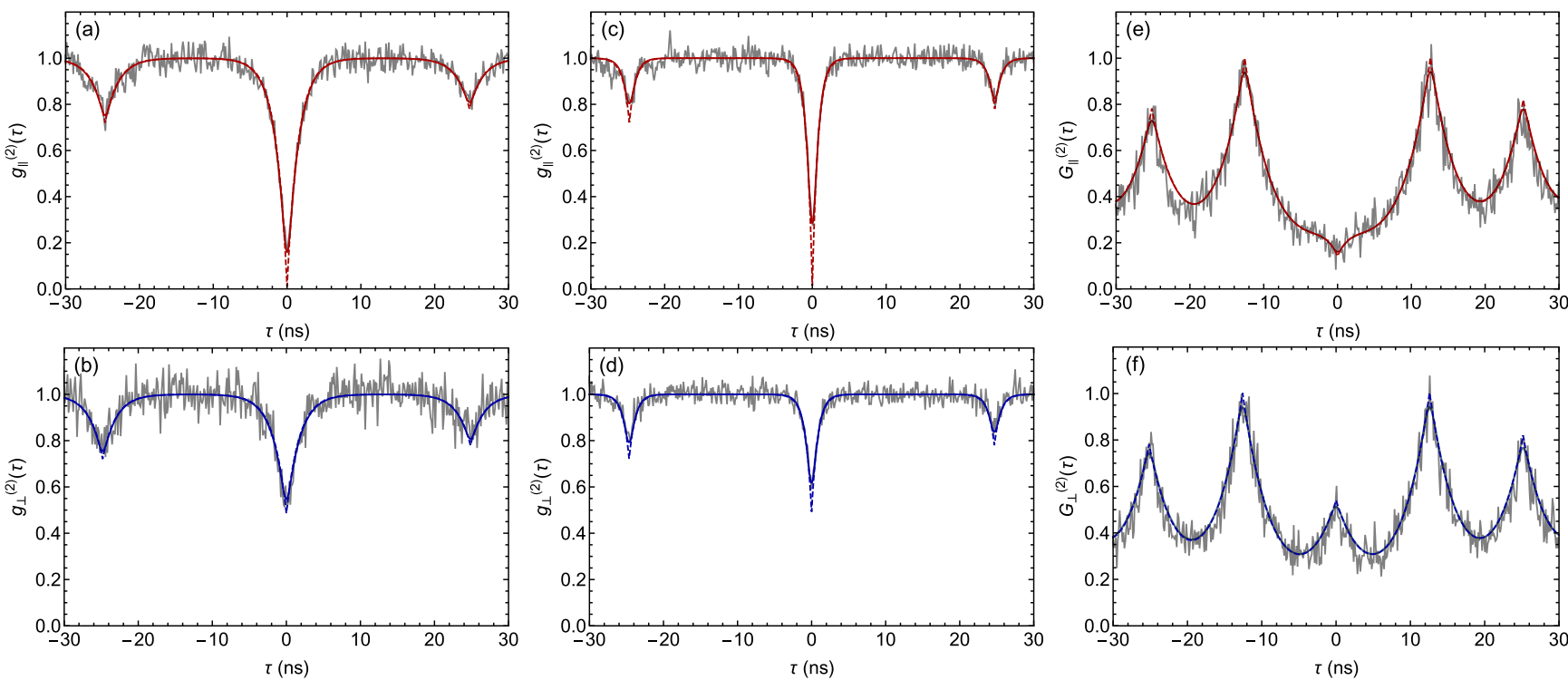

FIG. 3. Continuous wave (cw) and pulsed measurements of photon indistinguishability performed on the same molecule. Experimental data is in black, and colored curves show theoretical fits with (solid) and without (dashed) accounting for detector response. Measurements using cw excitation of (a) $g_{\|}^{(2)}(\tau)$ and (b) $g_{\perp}^{(2)}(\tau)$ at $S=1.3 \pm 0.1$ and of $\left(\right.$ c) $g_{\|}^{(2)}(\tau)$ and (d) $g_{\perp}^{(2)}(\tau)$ at $S=4.4 \pm 0.2$ with theoretical curves using Eq. (9). The effect of detector response on the narrow central feature in (c) is clearly visible. Pulsed excitation measurements of (e) $G_{\|}^{(2)}(\tau)$ and (f) $G_{\perp}^{(2)}(\tau)$ displaying anti-bunching and two-photon interference, with theory curves using Eq. (9), modified to account for the pulsed behavior. 


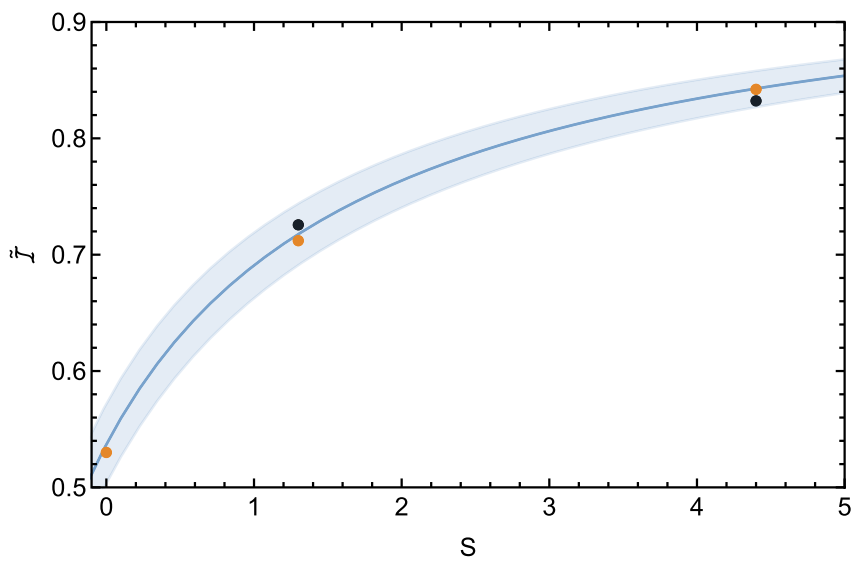

FIG. 4. Extracted $\tilde{\mathcal{I}}$ as a function of saturation parameter $(S)$. The prediction from Eq. (10) is shown as the solid line, with the shaded region indicating uncertainties in $\Gamma_{1}$ and $\Gamma_{2}$. Data points are from Eq. (10) using integration of the data (black) and fitted functions (orange). The data point at $S=0$ is from pulsed measurements in Figs. 3(e) and 3(f).

integral of the correlation function and as such does not affect the value for $\tilde{\mathcal{I}}$, we obtain using Eq. (7).

Figure 4 shows the ratio of the integrals described in Eq. (7) for measurements taken at $S=1.3 \pm 0.1$ and $S=4.4 \pm 0.2$; both values are well within the validity range for our model. Values of $\tilde{\mathcal{I}}$ based on the raw data (black) and the deconvolved functions (orange) are shown, and agree within error. Fitting Eq. (10) with $\mathcal{M}$ as the free variable gives $\mathcal{M}=0.96 \pm 0.01$ and an indistinguishability of $\mathcal{I}=0.53 \pm 0.01$ at $S=0$.

To confirm this result, we now turn to using pulsed excitation. We use a pulsed Ti:sapphire laser (Spectra-Physics, Tsunami) tuned to $766 \mathrm{~nm}$ and filtered to a bandwidth of $5 \mathrm{~nm}$ to excite the molecule again on a $S_{0,0} \rightarrow S_{1, n>0}$ transition. The parallel and perpendicular correlation functions are shown in Figs. 3(e) and 3(f), with each normalized to one. Here the $\sim 12.5 \mathrm{~ns}$ laser repetition period is only a few times longer than the $\sim 4$ ns lifetime of the molecule, and as such photons from subsequent pulses partially overlap. When taking the difference between the $G_{\| / \perp}^{(2)}(\tau)$ measurements in Eq. (4) contributions from the overlapping side features cancel, though this is not the case inthe denominator. This requires fitting to subtract the contribution of side features from the data to give the true integral of the central feature needed to quantify the indistinguishability according to Eq. (4). In doing so we find $\mathcal{I}=0.48 \pm 0.02$. This is lower than in the $\mathrm{cw}$ measurement due to imperfect temporal overlap arising from a mismatch of the fiber delay and the pulse repetition period in our interferometer. This can be accounted for with a correction factor of $e^{-\Gamma_{1} \Delta \tau}$ where $\Delta \tau$ is the time difference between the laser repetition period and the delay time from the fiber [33]. This is $0.91 \pm 0.02$ for our setup, and after this correction, we find $\mathcal{I}=0.53 \pm 0.02$, matching the value found through $\mathrm{cw}$ excitation. This is in line with the expected $\mathcal{I}$ value when considering $\mathcal{I}=\Gamma_{1} / 2 \Gamma_{2} \times \mathcal{M}=0.54 \pm 0.09$, where the polarization drift $(0.95)$ and branching ratio $(0.99)$ are contributing to $\mathcal{M}$. The indistinguishability is limited primarily by excess thermal dephasing, which greater cooling can eliminate [19,31]. Additionally, these measurements highlight the potential of single molecules for quantum technology applications [16] when considering their integration into nanophotonic structures such as waveguides [34,35], patterned polymers [36,37], and cavities [38].

\section{CONCLUSIONS}

In this work, we have shown a method to extract the full wave-packet indistinguishability of photons from a cwexcited single quantum emitter using two-photon interference measurements. This was experimentally verified by comparing photon indistinguishability found from $\mathrm{cw}$ and pulsed measurements performed on a single DBT molecule at cryogenic temperatures. Previous discussion of $\mathrm{cw}$ two-photon interference measurements has been limited to stating $g_{\| / \perp}^{(2)}(0)$ values, a metric that is not independent of the detector timing resolution and does not account for $S$. In this work, we provide a method for measuring indistinguishability of the full photon wave packet across all time, and functions describing measurements performed at $S \neq 0$. We note that our underlying theoretical treatment holds for other emitters and more complex systems. We already account for the coherent excitation to a third energy level and find a suitable parameter range for disregarding coherent effects and could be expanded to considering the effects of optical cavities on photon emission [14]. The interference of photons from two separate emitters has also been demonstrated with defects in diamond [39,40], quantum dots [41,42], and molecules [43]. Our method could be straightforwardly extended to account for the effects of driving on these systems, and could include further parameters such as different central frequencies and dephasing rates of the two emitters used.

In contrast to the pulsed case, determining indistinguishability from $\mathrm{cw}$ excitation requires multiple measurements at known pump powers, or a single measurement at a known $S$. However, it allows extraction of the indistinguishability from the raw data independently of the ratio of emitter lifetime to laser repetition rate. As such there is also no requirement for the interferometer delay to be a multiple of the laser repetition period, and $\mathrm{cw}$ excitation may also be more convenient due to the higher count rates and the greater spectral selectivity provided. These advantages open the possibility of performing multimode quantum interference experiments such as boson sampling [3] with a single cw-driven emitter and appropriate optical delay lines, thereby simplifying experimental demonstrations.

\section{ACKNOWLEDGMENTS}

We thank Jon Dyne and Dave Pitman for their expert mechanical workshop support. This work was supported by EPSRC (EP/P030130/1, EP/P01058X/1, EP/R044031/1, $\mathrm{EP} / \mathrm{S} 023607 / 1$, and EP/L015544/1), the Royal Society (UF160475), and the EraNET Cofund Initiative QuantERA under the European Unions Horizon 2020 research and innovation programme, Grant No. 731473 (ORQUID Project).

\section{APPENDIX A: INTERFERENCE THEORY}

We seek to derive the general second-order correlation function for the output fields of a two-photon interference 


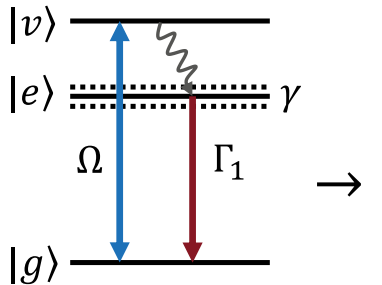

(a)

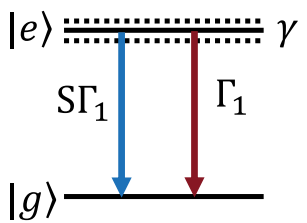

(b)
FIG. 5. (a) Schematic diagram of nonresonant driving from the ground $|g\rangle$ to a higher vibrational level $|v\rangle$, modelled by coherent driving with the Rabi frequency $\Omega$. The fast nonradiative decay rate from $|v\rangle \rightarrow|e\rangle$ is given by $\beta$. Spontaneous emission from the excited state $|e\rangle$ is given by $\Gamma_{1}$ and pure dephasing is given by $\gamma$. (b) Effective two level system by adiabatic elimination of the pump level, giving a driving rate $S \Gamma_{1}$ with the saturation parameter $\mathrm{S}$.

experiment. For this set up we have two (positive) input fields $E_{1}(t)$ and $E_{2}(t)$ which pass through a 50:50 beam splitter and are related to the (positive) detected fields $E_{3}(t)$ and $E_{4}(t)$ by $E_{3}(t)=\frac{1}{\sqrt{2}}\left(E_{1}(t)+E_{2}(t)\right)$ and $E_{4}(t)=\frac{1}{\sqrt{2}}\left(E_{2}(t)-\right.$ $\left.E_{1}(t)\right)$ [24]. The unnormalized general cross-correlation function for the output fields with parallel polarization between interferometer arms is

$$
G_{\|}^{(2)}(\tau, t)=\left\langle E_{3}^{\dagger}(t) E_{4}^{\dagger}(t+\tau) E_{4}(t+\tau) E_{3}(t)\right\rangle,
$$

where the output field $E_{3}$ is detected at $t$ and the output field $E_{4}$ is detected at $t+\tau$ leading us to define $\tau$ as the time delay between the two detection measurements. Substituting the input fields into Eq. (A1), we find

$$
\begin{aligned}
G_{\|}^{(2)}(\tau, t)= & \frac{1}{4}\left\langle\left(E_{1}^{\dagger}(t)+E_{2}^{\dagger}(t)\right)\left(E_{2}^{\dagger}(t+\tau)-E_{1}^{\dagger}(t+\tau)\right)\right. \\
& \left.\times\left(E_{2}(t+\tau)-E_{1}(t+\tau)\right)\left(E_{1}(t)+E_{2}(t)\right)\right\rangle .
\end{aligned}
$$

Simplifying Eq. (A2) as we assume $E_{1}$ and $E_{2}$ originate from the same emitter and are statistically independent; we therefore factorize and drop the numbered subscript. Expanding the correlation function in Eq. (A2) gives eight terms which are linear in $\langle E\rangle$ and two terms in the form $\langle E E\rangle$ which both go to zero, as expectation values linear in ladder operators are zero [25]. We find the general form

$$
\begin{aligned}
G_{\|}^{(2)}(\tau, t)= & \frac{1}{2}\left(\left\langle E^{\dagger}(t) E^{\dagger}(t+\tau) E(t+\tau) E(t)\right\rangle\right. \\
& +\left\langle E^{\dagger}(t) E(t)\right\rangle\left\langle E^{\dagger}(t+\tau) E(t+\tau)\right\rangle \\
& \left.-\|\left\langle E^{\dagger}(t+\tau) E(t)\right\rangle^{2}\right) .
\end{aligned}
$$

\section{APPENDIX B: ADIABATIC ELIMINATION}

To model nonresonant continuous wave excitation of a single emitter the three-level system shown in Fig. 5(a) is first considered. Defining the states $|v\rangle=(1,0,0),|e\rangle=$ $(0,1,0),|g\rangle=(0,0,1)$ and the operators $\sigma=|g\rangle\langle e|, \sigma_{v g}=$ $|v\rangle\langle g|$, and $\sigma_{e v}=|e\rangle\langle v|$. The subsequent Born-Markov second-order master equation to describe this system is

$$
\begin{aligned}
\partial_{t} \rho(t)= & -i\left[H_{\mathrm{S}}, \rho\right]+\Gamma_{1} \mathcal{L}_{\sigma}[\rho(t)] \\
& +\beta \mathcal{L}_{\sigma_{e v}}[\rho(t)]+2 \gamma \mathcal{L}_{\sigma^{\dagger} \sigma}[\rho(t)],
\end{aligned}
$$

with $H_{\mathrm{S}}=\Omega / 2\left(\sigma_{v g}+\sigma_{v g}^{\dagger}\right)$ representing the coherent driving with Rabi frequency $\Omega$.

Deriving an effective two-level system by adiabatically eliminating the higher order energy state, see Fig. 5. Starting with the optical Bloch equations for the three level nonresonantly driven system found from Eq. (B1), we find

$$
\begin{aligned}
& \dot{\rho}_{v v}(t)=\frac{i \Omega}{2}\left(\rho_{v g}(t)-\rho_{g v}(t)\right)-\beta \rho_{v v}(t), \\
& \dot{\rho}_{e e}(t)=-\Gamma_{1} \rho_{e e}(t)+\beta \rho_{v v}(t), \\
& \dot{\rho}_{g g}(t)=-\frac{i \Omega}{2}\left(\rho_{v g}(t)-\rho_{g v}(t)\right)+\Gamma_{1} \rho_{e e}(t), \\
& \dot{\rho}_{g v}(t)=\frac{i \Omega}{2}\left(\rho_{g g}(t)-\rho_{v v}(t)\right)-\frac{\beta}{2} \rho_{g v}(t), \\
& \dot{\rho}_{g e}(t)=-\frac{i \Omega}{2} \rho_{v e}(t)-\frac{\Gamma_{1}}{2} \rho_{g e}(t)-\gamma \rho_{g e}(t), \\
& \dot{\rho}_{v e}(t)=-\frac{i \Omega}{2} \rho_{g e}(t)-\frac{\Gamma_{1}}{2} \rho_{v e}(t)-\frac{\beta}{2} \rho_{v e}(t)-\gamma \rho_{v e}(t),
\end{aligned}
$$

where $\rho_{X Y}(t)=\langle X|\rho(t)| Y\rangle$ [44]. Solving firstly Eq. (B5) with an integrating factor to find

$$
\rho_{g v}(t)=\frac{i \Omega}{2} \int_{0}^{t} d t^{\prime} e^{-\frac{\beta}{2}\left(t-t^{\prime}\right)}\left(\rho_{g g}\left(t^{\prime}\right)-\rho_{v v}\left(t^{\prime}\right)\right),
$$

which can be solved for the case of $\beta \gg \Omega$ to give $\rho_{g v}(t) \approx$ $\frac{i \Omega}{\beta}\left(\rho_{g g}(t)-\rho_{v v}(t)\right)$, and by similar methodology $\rho_{v g}(t) \approx$ $-\frac{i \Omega}{\beta}\left(\rho_{g g}(t)-\rho_{v v}(t)\right)$. Using these forms for $\rho_{g v}(t)$ and $\rho_{v g}(t)$ and substituting into Eq. (B2), we find

$$
\dot{\rho}_{v v}(t)=-\frac{\Omega^{2}+\beta^{2}}{\beta} \rho_{v v}(t)+\frac{\Omega^{2}}{\beta} \rho_{g g}(t) .
$$

Solving Eq. (B9) using an integrating factor again we have

$$
\begin{aligned}
\rho_{v v}(t) & =\frac{\Omega^{2}}{\beta} \int_{0}^{t} e^{-\frac{\Omega^{2}+\beta^{2}}{\beta}\left(t-t^{\prime}\right)} \rho_{g g}\left(t^{\prime}\right) d t^{\prime} \\
& \approx \frac{\Omega^{2}}{\Omega^{2}+\beta^{2}} \rho_{g g}(t) .
\end{aligned}
$$

Finally, solving Eq. (B7) using the same methodology as above we find

$$
\begin{aligned}
\rho_{v e}(t) & =-\frac{i \Omega}{2} \int_{0}^{t} e^{-\left(\frac{\Gamma_{1}+\beta}{2}+\gamma\right)\left(t-t^{\prime}\right)} \rho_{g e}\left(t^{\prime}\right) d t^{\prime} \\
& \approx-i \frac{\Omega}{\beta+\Gamma_{1}+2 \gamma} \rho_{g e}(t) .
\end{aligned}
$$

Making a change of variables to the saturation parameter defined by $S=\Omega^{2} / \beta \Gamma_{1}$, we recover the ground and excited state optical Bloch equations for the effective two-level system

$$
\begin{aligned}
\dot{\rho}_{e e}(t) & =-\Gamma_{1} \rho_{e e}(t)+\beta \frac{\Omega^{2}}{\Omega^{2}+\beta^{2}} \rho_{g g}(t) \\
& \approx-\Gamma_{1} \rho_{e e}(t)+S \Gamma_{1} \rho_{g g}(t), \\
\dot{\rho}_{g g}(t) & \approx \Gamma_{1} \rho_{e e}(t)-S \Gamma_{1} \rho_{g g}(t),
\end{aligned}
$$

which holds as long as $\beta \gg \Omega$. We can further manipulate this equality as $\Omega=\sqrt{S \Gamma_{1} \beta}$, leading to the constraint $\beta \gg S \Gamma_{1}$. The final optical Bloch equation to consider is the $\dot{\rho}_{g e}(t)$ 
contribution. This leads to an interesting prefactor upon substitution of Eq. (B11) into Eq. (B6), we find

$$
\dot{\rho}_{g e}(t)=-\frac{S \Gamma_{1} \beta}{2\left(\beta+2 \Gamma_{2}\right)} \rho_{g e}(t)-\frac{\Gamma_{1}}{2} \rho_{g e}(t)-\gamma \rho_{g e}(t),
$$

which for $\beta \gg \Gamma_{2}$ can be simplified to recover the two-level system optical Bloch equation

$$
\dot{\rho}_{g e}(t)=-\frac{S \Gamma_{1}}{2} \rho_{g e}(t)-\frac{\Gamma_{1}}{2} \rho_{g e}(t)-\gamma \rho_{g e}(t),
$$

which can be represented as a Born-Markov master equation as

$$
\partial_{t} \rho(t)=\Gamma_{1}\left(\mathcal{L}_{\sigma}[\rho(t)]+S \mathcal{L}_{\sigma^{\dagger}}[\rho(t)]\right)+2 \gamma \mathcal{L}_{\sigma_{e e}}[\rho(t)],
$$

where $\quad \mathcal{L}_{X}[\rho(t)]=X \rho(t) X^{\dagger}-\frac{1}{2}\left\{X^{\dagger} X, \rho(t)\right\} \quad$ is a Lindblad operator which captures open quantum system dissipators.
[1] E. Knill, R. Laflamme, and G. J. Milburn, A scheme for efficient quantum computation with linear optics, Nature 409, 46 (2001).

[2] B. Bell, A. S. Clark, M. S. Tame, M. Halder, J. Fulconis, W. J. Wadsworth, and J. G. Rarity, Experimental characterization of photonic fusion using fiber sources, New J. Phys. 14, 023021 (2012).

[3] M. Bentivegna, N. Spagnolo, C. Vitelli, F. Flamini, N. Viggianiello, L. Latmiral, P. Mataloni, D. J. Brod, E. F. Galvão, A. Crespi, R. Ramponi, R. Osellame, and F. Sciarrino, Experimental scattershot boson sampling, Sci. Adv. 1, e1400255 (2015).

[4] V. Giovannetti, S. Lloyd, and L. Maccone, Advances in quantum metrology, Nat. Photon. 5, 222 (2011).

[5] H. de Riedmatten, I. Marcikic, W. Tittel, H. Zbinden, D. Collins, and N. Gisin, Long Distance Quantum Teleportation in a Quantum Relay Configuration, Phys. Rev. Lett. 92, 047904 (2004).

[6] D. Llewellyn, Y. Ding, I. I. Faruque, S. Paesani, D. Bacco, R. Santagati, Y.-J. Qian, Y. Li, Y.-F. Xiao, M. Huber, M. Malik, G. F. Sinclair, X. Zhou, K. Rottwitt, J. L. OBrien, J. G. Rarity, Q. Gong, L. K. Oxenlowe, J. Wang, and M. G. Thompson, Chipto-chip quantum teleportation and multi-photon entanglement in silicon, Nat. Phys. 16, 148 (2020).

[7] R. Ghosh and L. Mandel, Observation of Nonclassical Effects in the Interference of Two Photons, Phys. Rev. Lett. 59, 1903 (1987).

[8] C. K. Hong, Z. Y. Ou, and L. Mandel, Measurement of Subpicosecond Time Intervals Between Two Photons by Interference, Phys. Rev. Lett. 59, 2044 (1987).

[9] M. D. Eisaman, J. Fan, A. Migdall, and S. V. Polyakov, Invited Review Article: Single-photon sources and detectors, Rev. Sci. Instrum. 82, 071101 (2011).

[10] M. Hijlkema, B. Weber, H. P. Specht, S. C. Webster, A. Kuhn, and G. Rempe, A single-photon server with just one atom, Nat. Phys. 3, 253 (2007).

[11] C. Santori, D. Fattal, J. Vučković, G. S. Solomon, and Y. Yamamoto, Indistinguishable photons from a single-photon device, Nature 419, 594 (2002).

[12] N. Somaschi, V. Giesz, L. De Santis, J. C. Loredo, M. P. Almeida, G. Hornecker, S. L. Portalupi, T. Grange, C. Antón, J. Demory, C. Gómez, I. Sagnes, N. D. Lanzillotti-Kimura, A. Lemaítre, A. Auffeves, A. G. White, L. Lanco, and P. Senellart, Near-optimal single-photon sources in the solid state, Nat. Photon. 10, 340 (2016).

[13] H. Wang, Y. M. He, T. H. Chung, H. Hu, Y. Yu, S. Chen, X. Ding, M. C. Chen, J. Qin, X. Yang, R. Z. Liu, Z. C. Duan, J. P. Li, S. Gerhardt, K. Winkler, J. Jurkat, L. J. Wang, N. Gregersen,
Y. H. Huo, Q. Dai et al., Towards optimal single-photon sources from polarized microcavities, Nat. Photon. 13, 770 (2019).

[14] J. Iles-Smith, D. P. S. McCutcheon, A. Nazir, and J. Mørk, Phonon scattering inhibits simultaneous near-unity efficiency and indistinguishability in semiconductor single-photon sources, Nat. Photon. 11, 521 (2017).

[15] I. Aharonovich, S. Castelletto, D. A. Simpson, C.-H. Su, A. D. Greentree, and S. Prawer, Diamond-based single-photon emitters, Rep. Prog. Phys. 74, 076501 (2011).

[16] C. Toninelli, I. Gerhardt, A. S. Clark, A. Reserbat-Plantey, S. Götzinger, Z. Ristanovic, M. Colautti, P. Lombardi, K. D. Major, I. Deperasińska, W. H. Pernice, F. H. L. Koppens, B. Kozankiewicz, A. Gourdon, V. Sandoghdar, and M. Orrit, Single organic molecules for photonic quantum technologies, Nat. Mater. 20, 1615 (2021).

[17] N. Tomm, A. Javadi, N. O. Antoniadis, D. Najer, M. C. Löbl, A. R. Korsch, R. Schott, S. R. Valentin, A. D. Wieck, A. Ludwig, and R. J. Warburton, A bright and fast source of coherent single photons, Nat. Nanotechnol. 16, 399 (2021).

[18] R. B. Patel, A. J. Bennett, K. Cooper, P. Atkinson, C. A. Nicoll, D. A. Ritchie, and A. J. Shields, Postselective Two-Photon Interference from a Continuous Nonclassical Stream of Photons Emitted by a Quantum Dot, Phys. Rev. Lett. 100, 207405 (2008).

[19] J. B. Trebbia, P. Tamarat, and B. Lounis, Indistinguishable nearinfrared single photons from an individual organic molecule, Phys. Rev. A 82, 063803 (2010).

[20] M. Rezai, J. Wrachtrup, and I. Gerhardt, Coherence Properties of Molecular Single Photons for Quantum Networks, Phys. Rev. X 8, 031026 (2018).

[21] P. Lombardi, M. Colautti, R. Duquennoy, G. Murtaza, P. Majumder, and C. Toninelli, Triggered emission of indistinguishable photons from an organic dye molecule, Appl. Phys. Lett. 118, 204002 (2021).

[22] J. Iles-Smith, D. P. S. McCutcheon, J. Mørk, and A. Nazir, Limits to coherent scattering and photon coalescence from solid-state quantum emitters, Phys. Rev. B 95, 201305(R) (2017).

[23] R. Proux, M. Maragkou, E. Baudin, C. Voisin, P. Roussignol, and C. Diederichs, Measuring the Photon Coalescence Time Window in the Continuous-Wave Regime for Resonantly Driven Semiconductor Quantum Dots, Phys. Rev. Lett. 114, 067401 (2015).

[24] A. Kiraz, M. Atatüre, and A. Imamoğlu, Quantum-dot singlephoton sources: Prospects for applications in linear optics quantum-information processing, Phys. Rev. A 69, 032305(R) (2004). 
[25] S. Unsleber, D. P. S. McCutcheon, M. Dambach, M. Lermer, N. Gregersen, S. Höfling, J. Mørk, C. Schneider, and M. Kamp, Two-photon interference from a quantum dot microcavity: Persistent pure dephasing and suppression of time jitter, Phys. Rev. B 91, 075413 (2015).

[26] J. Bylander, I. Robert-Philip, and I. Abram, Interference and correlation of two independent photons, Eur. Phys. J. D 22, 295 (2003).

[27] G. Guarnieri, A. Smirne, and B. Vacchini, Quantum regression theorem and non-Markovianity of quantum dynamics, Phys. Rev. A 90, 022110 (2014).

[28] See Supplemental Material at http://link.aps.org/supplemental/ 10.1103/PhysRevResearch.4.013037 for [give brief description of material].

[29] R. C. Schofield, K. D. Major, S. Grandi, S. Boissier, E. A. Hinds, and A. S. Clark, Efficient excitation of dye molecules for single photon generation, J. Phys. Commun. 2, 115027 (2018).

[30] S. Pazzagli, P. Lombardi, D. Martella, M. Colautti, B. Tiribilli, F. S. Cataliotti, and C. Toninelli, Self-assembled nanocrystals of polycyclic aromatic hydrocarbons show photostable singlephoton emission, ACS Nano 12, 4295 (2018).

[31] C. Clear, R. C. Schofield, K. D. Major, J. Iles-Smith, A. S. Clark, and D. P. S. McCutcheon, Phonon-Induced Optical Dephasing in Single Organic Molecules, Phys. Rev. Lett. 124, 153602 (2020).

[32] S. Grandi, K. D. Major, C. Polisseni, S. Boissier, A. S. Clark, and E. A. Hinds, Quantum dynamics of a driven two-level molecule with variable dephasing, Phys. Rev. A 94, 063839 (2016).

[33] C. Schneider, P. Gold, C.-Y. Lu, S. Höfling, J.-W. Pan, and M. Kamp, Single semiconductor quantum dots in microcavities: Bright sources of indistinguishable photons, in Engineering the Atom-Photon Interaction: Controlling Fundamental Processes with Photons, Atoms and Solids, edited by A. Predojević and M. W. Mitchell (Springer International Publishing, Cham, 2015), pp. 343-361

[34] S. Boissier, R. C. Schofield, L. Jin, A. Ovvyan, S. Nur, F. H. L. Koppens, C. Toninelli, W. H. P. Pernice, K. D. Major, E. A. Hinds, and A. S. Clark, Coherent characterisation of a single molecule in a photonic black box, Nat. Commun. 12, 706 (2021).
[35] D. Rattenbacher, A. Shkarin, J. Renger, T. Utikal, S. Götzinger, and V. Sandoghdar, Coherent coupling of single molecules to on-chip ring resonators, New J. Phys. 21, 062002 (2019).

[36] C. Ciancico, K. G. Schädler, S. Pazzagli, M. Colautti, P. Lombardi, J. Osmond, C. Dore, A. Mihi, A. P. Ovvyan, W. H. Pernice, E. Berretti, A. Lavacchi, C. Toninelli, F. H. Koppens, and A. Reserbat-Plantey, Narrow line width quantum emitters in an electron-beam-shaped polymer, ACS Photonics 6, 3120 (2019).

[37] R. C. Schofield, D. P. Bogusz, R. A. Hoggarth, S. Nur, K. D. Major, and A. S. Clark, Polymer-encapsulated organic nanocrystals for single photon emission, Opt. Mater. Express 10, 1586 (2020).

[38] D. Wang, H. Kelkar, D. Martin-Cano, D. Rattenbacher, A Shkarin, T. Utikal, S. Götzinger, and V. Sandoghdar, Turning a molecule into a coherent two-level quantum system, Nat. Phys. 15, 483 (2019).

[39] A. Sipahigil, K. D. Jahnke, L. J. Rogers, T. Teraji, J. Isoya, A. S. Zibrov, F. Jelezko, and M. D. Lukin, Indistinguishable Photons from Separated Silicon-Vacancy Centers in Diamond, Phys. Rev. Lett. 113, 113602 (2014).

[40] H. Bernien, L. Childress, L. Robledo, M. Markham, D. Twitchen, and R. Hanson, Two-Photon Quantum Interference from Separate Nitrogen Vacancy Centers in Diamond, Phys. Rev. Lett. 108, 043604 (2012).

[41] E. B. Flagg, A. Muller, S. V. Polyakov, A. Ling, A. Migdall, and G. S. Solomon, Interference of Single Photons from Two Separate Semiconductor Quantum Dots, Phys. Rev. Lett. 104, 137401 (2010).

[42] R. B. Patel, A. J. Bennett, I. Farrer, C. A. Nicoll, D. A. Ritchie, and A. J. Shields, Two-photon interference of the emission from electrically tunable remote quantum dots, Nat. Photon. 4, 632 (2010).

[43] R. Lettow, Y. L. A. Rezus, A. Renn, G. Zumofen, E. Ikonen, S. Götzinger, and V. Sandoghdar, Quantum Interference of Tunably Indistinguishable Photons from Remote Organic Molecules, Phys. Rev. Lett. 104, 123605 (2010).

[44] G. J. De Valcárcel, E. Roldán, and F. Prati, Semiclassical theory of amplification and lasing, Revista Mexicana de Física E 52, 198 (2006). 